\title{
The category of equilogical spaces and the effective topos as homotopical quotients
}

\author{
Giuseppe Rosolini ${ }^{1}$
}

Received: 30 April 2015 / Accepted: 23 November 2015 / Published online: 25 October 2016

(C) Tbilisi Centre for Mathematical Sciences 2016

\begin{abstract}
We show that the two models of an extensional version of Martin-Löf type theory, those given by the category of equilogical spaces and by the effective topos, are homotopical quotients of appropriate categories of 2-groupoids.
\end{abstract}

\section{Introduction}

The category of $\mathrm{T}_{0}$-spaces embeds fully in the category of equilogical spaces; the category of equilogical spaces is locally cartesian closed and the embedding functor preserves products and any exponential available in the original category. Thus the category of equilogical spaces provides a nice extension of the category of $\mathrm{T}_{0}$-spaces. The effective topos is the categorical rendering of Kleene's realizability model for intuitionistic logic, and is the first interesting example of a non-Grothendieck topos. We show that the category of equilogical spaces is the homotopical quotient of a category of groupoids, and that the effective topos is the homotopical quotient of a category of 2-groupoids of partitioned assemblies.

Groupoids are a main tool in algebraic topology, see [4] and groupoids were the firstnontrivial models of the intensional version of Martin-Löf Type Theory in [12].

Dedicated to Ronald Brown on the occasion of his 80th birthday.

Communicated by Tim Porter and George Janelidze.

Projects MIUR-PRIN 2010-2011 and Correctness by Construction (EU 7th framework programme, grant no. PIRSES-GA-2013-612638) provided support for the research presented in the paper.

$\bowtie$ Giuseppe Rosolini

rosolini@unige.it

1 DIMA, via Dodecaneso 35, 16146 Genoa, Italy 
Moreover in recent years the Univalent Foundations Program, see [19], has advocated a strong connection between algebraic topology and type theory.

Since both the category of equilogical spaces and the effective topos are models of an extensional version of Martin-Löf type theory, it is useful to find that each comes from the "extensionalization" of a model of intensional type theory and that such a process is actually a homotopical quotient. We should stop here to point out that the meaning we adopt for an homotopical quotient of a category is in line with a suggestion in [8] and is the more naive notion obtained from an interval-like object than that derived from a Quillen model category - the main reason is that one of the two example categories we study is neither complete nor cocomplete. So, as a homotopical quotient, we shall consider a category obtained as a quotient category from a category $C$ with finite limits, as follows:

- there is a fixed interval-like object $I$, i.e. it has two global points $0: T \longrightarrow I$ and $1: T \longrightarrow I$ whose pushout

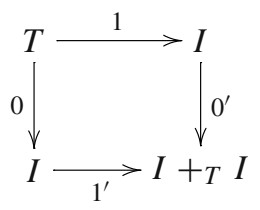

exists in $C$ and is stable under products, an arrow $\gamma: I \longrightarrow I+{ }_{T} I$ and an arrow $\iota: I \longrightarrow I$ such that the four arrows together with the unique arrow $!: I \longrightarrow T$ form an equivalence co-span in $\mathcal{C}$, i.e. the following diagrams commute
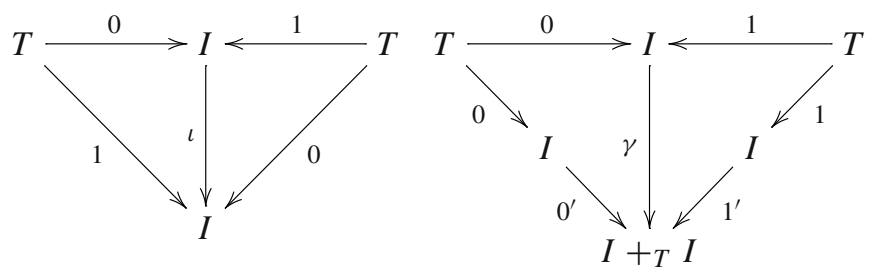

—note that there is also a necessarily commutative diagram

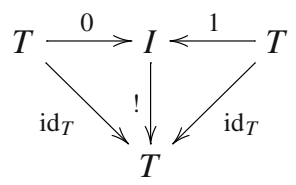

since $T$ is terminal-; 
- two arrows $f, g: X \longrightarrow Y$ are identified in the quotient if there is an arrow $h: X \times I \longrightarrow Y$ such that the following diagram commute

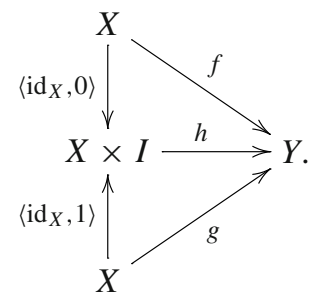

The condition of the structure on $I$ ensures that the identification is an equivalence relation on parallel arrows in $C$.

It seems plausible that the categories we analyse in the following sustain suitable notions of fibrations, cofibrations and weak equivalences - in particular, that a map of the kind $\left\langle\operatorname{id}_{X}, i\right\rangle: X \longrightarrow X \times I, i=0,1$, is a weak equivalence. But the categories are certainly not complete, nor cocomplete, and that prevents a direct comparison with standard homotopical quotients. It will be considered in future work.

We introduce the category of equilogical spaces in Sect. 2 and we recall one of the presentations of the effective topos in Sect. 3, reviewing properties which are needed in the following sections. In Sect. 4 we determine a category $\mathcal{A}$ of topological groupoids and an interval-like topological groupoid II such that the homotopical quotient of $\mathcal{A}$ determined by $\mathbb{I}$ is equivalent to the category of equilogical spaces. In Sect. 5 we produce a similar result for the effective topos using a category of 2-groupoids on partitioned assemblies.

The idea of the paper grew out of work on models for Homotopy Type Theory during discussions at CMU with Steve Awodey and the members of the lively HoTT group there. The author acknowledges how the stimulating environment helped develop the ideas presented in the paper and warmly thanks all the participants for their strong support.

The final draft of the paper was prepared following some interesting remarks made by an anonymous referee; the author thankfully acknowledges the referee's unconditional contribution.

\section{Equilogical spaces}

Recall from [1,18] that an equilogical space $\mathcal{E}=\left(S_{\mathcal{E}}, \tau_{\mathcal{E}}, \sim_{\mathcal{E}}\right)$ consists of a $\mathrm{T}_{0}$-space $\left(S_{\mathcal{E}}, \tau_{\mathcal{E}}\right)$ and an equivalence relation $\sim_{\mathcal{E}} \subseteq S_{\mathcal{E}} \times S_{\mathcal{E}}$ on the points of the space.

A map $[f]: \mathcal{E} \longrightarrow \mathcal{F}$ of equilogical spaces is an equivalence class of continuous functions $f:\left(S_{\mathcal{E}}, \tau_{\mathcal{E}}\right) \longrightarrow\left(S_{\mathcal{F}}, \tau_{\mathcal{F}}\right)$ preserving the equivalence relations, i.e. if $x \sim_{\mathcal{E}} x^{\prime}$, then $f(x) \sim_{\mathcal{F}} f\left(x^{\prime}\right)$ for all $x$ and $x^{\prime}$ in $S_{\mathcal{E}}$. For two such continuous functions $f, g:\left(S_{\mathcal{E}}, \tau_{\mathcal{E}}\right) \longrightarrow\left(S_{\mathcal{F}}, \tau_{\mathcal{F}}\right)$, one sets $f$ equivalent to $g$ when $f(x) \sim_{\mathcal{F}} g(x)$ for all $x \in S_{\mathcal{E}}$.

Composition of maps of equilogical spaces $[f]: \mathcal{E} \longrightarrow \mathcal{F}$ and $[g]: \mathcal{F} \longrightarrow \mathcal{G}$ is given on (any of) their continuous representatives: $[g] \circ[f]:=[g \circ f]$. 
The data above determine a category $\mathcal{E} q u$ of equilogical spaces. There is a full embedding

$$
Y: \mathcal{T}_{o p_{0}} \underset{\text { full }}{\longrightarrow} \mathcal{E} q u
$$

which maps a $\mathrm{T}_{0}$-space $(S, \tau)$ to the equilogical space on $(S, \tau)$ with the diagonal relation, i.e. the equilogical space $\left(S, \tau,=_{S}\right)$.

The category $\mathcal{E} q u$ is a locally cartesian closed full extension of the category $\mathcal{T}_{o} p_{0}$ of $\mathrm{T}_{0}$-spaces. In fact, it is the intersection of two other locally cartesian closed full extensions of

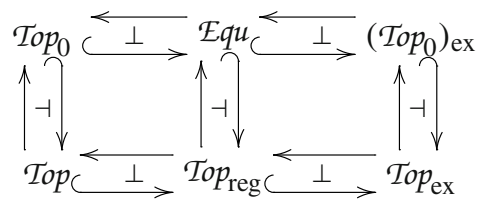

The exact completions $\left(\mathcal{T}_{o p_{0}}\right)_{\mathrm{ex}}$ and $\mathcal{T}_{o p_{\mathrm{ex}}}$ are pretoposes, while the regular completion $\mathcal{T o p}_{\text {reg }}$ is a quasitopos, see [15].

The product of equilogical spaces $\mathcal{E} \times \mathcal{F}$ is computed as expected taking the topological product $\left(S_{\mathcal{E}}, \tau_{\mathcal{E}}\right) \times\left(S_{\mathcal{F}}, \tau_{\mathcal{F}}\right)$ and the equivalence relation

$$
\langle a, b\rangle \sim_{\mathcal{E} \times \mathcal{F}}\left\langle a^{\prime}, b^{\prime}\right\rangle \quad \text { when } a \sim_{\mathcal{E}} a^{\prime} \text { and } b \sim_{\mathcal{F}} b^{\prime} .
$$

The projections to the factors are obvious.

The construction of the exponential $\mathcal{F}^{\mathcal{E}}$ is less direct and we refer the reader to the basic sources $[1,17,18]$ as well as $[2,3]$.

It is useful for the purpose of this paper to point out the strong similarity between the presentation of $\mathcal{E} q u$ and that of $\left(\mathcal{T}_{o p_{0}}\right)_{\mathrm{ex}}$. So recall from $[5,6,8,9]$ that the exact completion $\mathcal{C}_{\mathrm{ex}}$ of a category $\mathcal{C}$ with finite limits is a quotient category of the full subcategory $\operatorname{ES}(C)$ of the category $\stackrel{\vec{C}}{\rightarrow}$ of graphs in $C$ on the equivalence spans.

Recall that a (directed) graph in $C$ is a parallel pair $A_{1} \underset{d_{2}}{\stackrel{d_{1}}{\longrightarrow}} A_{0}$ of arrows of $C$ and a homomorphism from the graph $A_{1} \underset{d_{2}}{\stackrel{d_{1}}{\longrightarrow}} A_{0}$ to the graph $B_{1} \stackrel{e_{1}}{\longrightarrow} B_{0}$ is a pair $\left(f_{1}: A_{1} \longrightarrow B_{1}, f_{0}: A_{0} \longrightarrow B_{0}\right)$ of arrows in $C$ such that the following diagram commutes

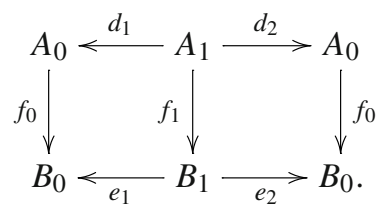


An equivalence span is a graph $A_{1} \underset{d_{2}}{\stackrel{d_{1}}{\longrightarrow}} A_{0}$ in $C$ which is reflexive, symmetric, and endowed with a compatible operation on pairs of consecutive arcs, i.e. there are arrows $r: A_{0} \longrightarrow A_{1}, s: A_{1} \longrightarrow A_{1}$, and $t: A_{1} \times A_{0} A_{1} \longrightarrow A_{1}$, where

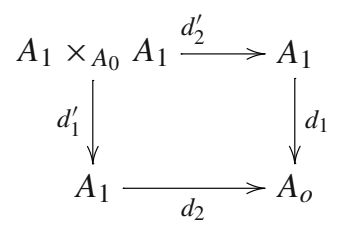

is a pullback in $\mathcal{C}$, such that the following diagrams commute:

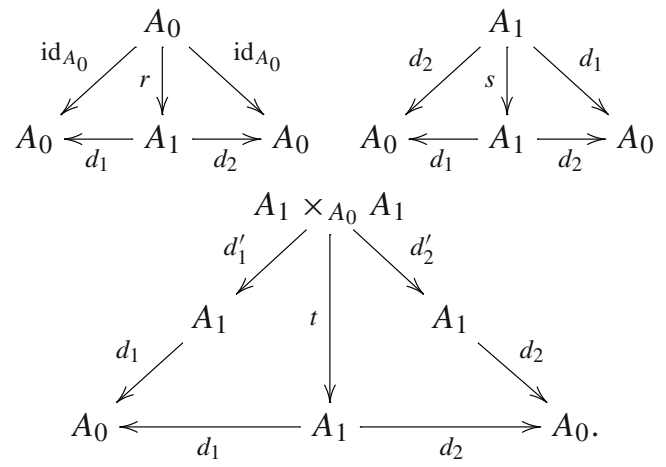

The quotient category $C_{\mathrm{ex}}$ is obtained by identifying homomorphisms $\left(f_{1}, f_{0}\right)$ and $\left(g_{1}, g_{0}\right)$ from $A_{1} \underset{d_{2}}{\stackrel{d_{1}}{\longrightarrow}} A_{0}$ to $B_{1} \underset{e_{2}}{\stackrel{e_{1}}{\longrightarrow}} B_{0}$ if there is an arrow $h: A_{0} \longrightarrow B_{1}$ such that

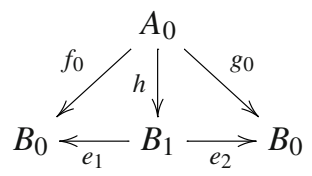

—nothing is asked of the other component.

The following proposition makes the similarity explicit.

Proposition 1 The category $\mathcal{E} q u$ is equivalent to the full subcategory $\mathcal{A}$ of $\left(\mathcal{T}_{o} p_{0}\right)_{\mathrm{ex}}$ on those equivalence spans $A_{1} \underset{d_{2}}{\stackrel{d_{1}}{\longrightarrow}} \longrightarrow A_{0}$ of topological spaces and continuous maps such that the pair $\left\langle d_{1}, d_{2}\right\rangle: A_{1} \longrightarrow A_{0} \times A_{0}$ is a subspace inclusion. 
Proof Consider an equivalence span $A=A_{1} \underset{d_{2}}{\stackrel{d_{1}}{\longrightarrow}} A_{0}$ of topological spaces and continuous maps such that the pair $\left\langle d_{1}, d_{2}\right\rangle: A_{1} \longrightarrow A_{0} \times A_{0}$ is a subspace inclusion. Note that the functions $r, s$ and $t$ requested by the definition of equivalence span are unique, and determine that the subset $\left|A_{1}\right|$ of pairs of points of $\left|A_{0}\right|$ is an equivalence relation. Write $F(A)$ for the equilogical space which consists of the topological space $A_{0}$ and the equivalence relation $\left|A_{1}\right|$.

For a homomorphism $\left(f_{1}, f_{0}\right)$ between two such equivalence spans, the component $f_{1}$ is uniquely determined by the other data as the restriction of the pair $\left\langle f_{0}, f_{0}\right\rangle$, and ensures that $f_{0}$ is a representative of a map of equilogical spaces. Moreover, in the quotient category $\left(\mathcal{T}_{o} p_{0}\right)_{\mathrm{ex}}$, the homomorphism $\left(f_{1}, f_{0}\right)$ is identified with $\left(g_{1}, g_{0}\right)$ precisely when $\langle f(x), g(x)\rangle$ is in $A_{1}$ for all points $x$ in $A_{0}$.

Thus the assignment $F\left(\left[f_{1}, f_{0}\right]\right)=\left[f_{0}\right]$ is well defined, and determines a functor from $\mathcal{A}$ to $\mathcal{E} q u$ which is full and faithful.

To see that $F$ is also bijective on objects, suppose $\mathcal{E}=\left(S_{\mathcal{E}}, \tau_{\mathcal{E}}, \sim_{\mathcal{E}}\right)$ is an equilogical space. Consider the subspace topology $\sigma_{\mathcal{E}}$ on $\sim_{\mathcal{E}} \subseteq S_{\mathcal{E}} \times S_{\mathcal{E}}$ and the graph of topological spaces

$$
\left(\sim_{\mathcal{E}}, \sigma_{\mathcal{E}}\right) \underset{\pi_{2}}{\stackrel{\pi_{1}}{\longrightarrow}}\left(S_{\mathcal{E}}, \tau_{\mathcal{E}}\right)
$$

induced by the two projections. It is easy to check that it is an equivalence span and, by construction, the pair $\left\langle\pi_{1}, \pi_{2}\right\rangle:\left(\sim_{\mathcal{E}}, \sigma_{\mathcal{E}}\right) \longrightarrow\left(S_{\mathcal{E}}, \tau_{\mathcal{E}}\right) \times\left(S_{\mathcal{E}}, \tau_{\mathcal{E}}\right)$ is a subspace inclusion. It is obvious that the functor $F$ takes that equivalence span of $\mathcal{A}$ to the equilogical space $\mathcal{E}$.

In the following, we shall refer to an equivalence span $A_{1} \underset{d_{2}}{\stackrel{d_{1}}{\longrightarrow}} A_{0}$ of topological spaces and continuous maps such that the pair $\left\langle d_{1}, d_{2}\right\rangle: A_{1} \longrightarrow A_{0}$ is a subspace inclusion as a subspatial equivalence span.

\section{The effective topos}

The effective topos Eff was introduced in [10,13]. It was shown in [16] that Eff is (equivalent to) the exact completion of the category $\mathcal{P} \mathcal{A} s m$ of partitioned assemblies, see [7].

A partitioned assembly is a function $\xi: X \longrightarrow \mathbb{N}$; a map $\begin{gathered}X \\ \downarrow \\ \mathbb{N}\end{gathered} \begin{array}{r}Y \\ \rightarrow \zeta \underset{\mathbb{N}}{\downarrow} \text { of }\end{array}$

partitioned assemblies is a function $f: X \longrightarrow Y$ such that there is a partial recursive function $\phi: \mathbb{N} \rightarrow \mathbb{N}$ such that the following diagram commutes 


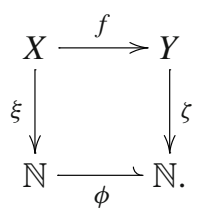

In order to make sure that the exact completion introduced in Sect. 2 can be applied to the category $\mathcal{P} \mathcal{A} s m$ we recall how finite limits can be obtained in that category.

The product of two partitioned assemblies is obtained by adopting some particular recursive enconding $\langle\langle n, m\rangle\rangle$ of pairs of numbers; the product partitioned assembly of X $\quad Y$

$\xi \downarrow$ and $\zeta \downarrow$ is the function

$\mathbb{N} \quad \mathbb{N}$

$$
(x, y) \mapsto\langle\langle\xi(x), \zeta(y)\rangle\rangle: X \times Y \longrightarrow \mathbb{N}
$$

with obvious projections.

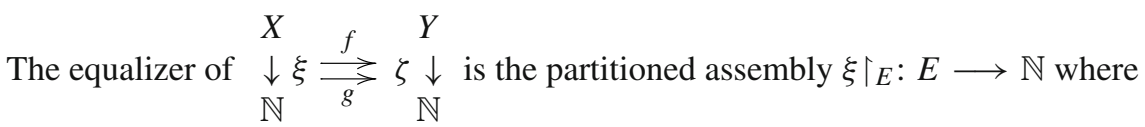
$E:=\{x \in \mathbb{N} \mid f(x)=g(x)\}$ with the obvious inclusion into $\xi \downarrow$.

The next result will be useful in the following.

Lemma 1 Every equivalence span

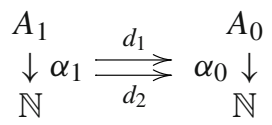

in $\mathcal{P} \mathcal{A s m}_{\mathrm{ex}}$ is isomorphic to one of the form

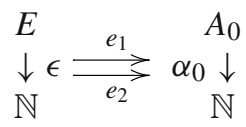

such that the triple $\left\langle e_{1}, e_{2}, \epsilon\right\rangle$ is monic.

Proof Consider an arbitrary equivalence span

$$
\begin{array}{ll}
A_{1} \\
\underset{\mathbb{N}}{\downarrow} \alpha_{1} \underset{d_{2}}{\stackrel{d_{1}}{\longrightarrow}} \underset{\mathbb{N}}{\longrightarrow} \alpha_{0} \underset{\downarrow}{\downarrow}
\end{array}
$$


in $\mathcal{P} \mathcal{A s m}_{\mathrm{ex}}$. So there are two partial recursive functions $\phi_{1}$ and $\phi_{2}$ such that the following diagram commutes

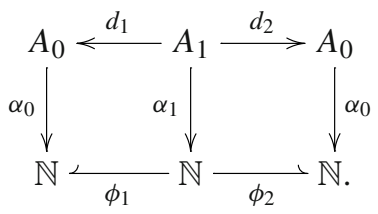

Take $E$ to be the image of the function $\left\langle d_{1}, d_{2}, \alpha_{1}\right\rangle: A_{1} \longrightarrow A_{0} \times A_{0} \times \mathbb{N}$, let $f: A_{1} \longrightarrow E$ be the factoring surjection, and let $\epsilon:=\pi_{3} \uparrow_{E}: E \longrightarrow \mathbb{N}$. Let E $\quad A_{0}$

$e_{1}, e_{2}: \downarrow \epsilon \longrightarrow \alpha_{0} \downarrow$ be the first and second projection respectively. It is easy to see $\mathbb{N} \quad \mathbb{N}$

that it is an equivalence span.

Clearly $f$ gives rise to a map of partitioned assemplies $\begin{aligned} & A_{1} \\ & \underset{\mathbb{N}}{\downarrow} \alpha_{1} \stackrel{f}{\longrightarrow} \in \downarrow \\ & \mathbb{N}\end{aligned}$ is a commutative diagram

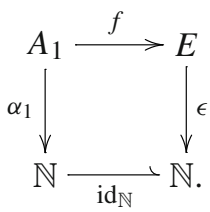

Moreover any section $s: E \longrightarrow A_{1}$ of $f$ (as a function of sets) is a map of

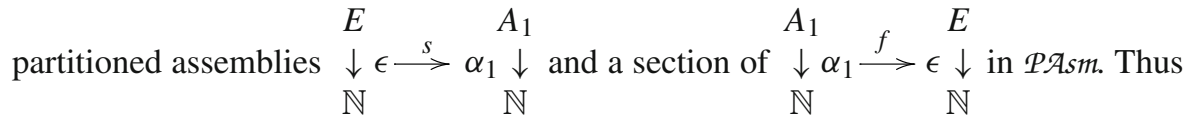
an appeal to the axiom of choice yields the conclusion.

Remark 1 Note that the axiom of choice was used in a crucial way in 1 to determine an equivalence span of the required form and the requested isomorphism, but the proof that

$$
\begin{array}{ll}
E & \stackrel{e_{1}}{\downarrow} \underset{e_{2}}{\longrightarrow} \underset{\mathbb{N}}{\longrightarrow} \\
\alpha_{0} & A_{0} \\
\mathbb{N}
\end{array}
$$

is an equivalence span does not require the use of the axiom of choice.

We conclude this brief review of the effective topos recalling a diagram of functors considered by Aurelio Carboni in [5] which shows how similar the situation is to that of topological spaces. Write $\mathcal{P} \mathcal{A}_{s m_{0}}$ for the full subcategory of $\mathcal{P} \mathcal{A} s m$ on those partitioned assemblies which are 1-1 (functions). This is clearly equivalent to the category $\mathcal{P R}$ 
whose objects are subsets of $\mathbb{N}$ and whose arrows are restriction of partial recursive functions between those, total on the domain.

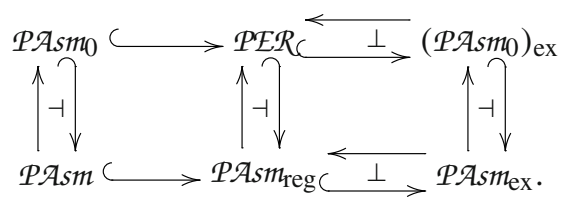

In the diagram of full subcategories of $\mathcal{E} f f$, the exact completion $\mathcal{P} \mathcal{A} s m_{\mathrm{ex}}$ is itself the effective topos; $\mathcal{P} \mathcal{A} s m_{\text {reg }}$ is the full subcategory of $\mathcal{E} f f$ on the $\neg \neg$-separated objects; $(\mathcal{P A s m})_{\mathrm{ex}}$ is the full subcategory of $\mathcal{E} f f$ on the discrete objects-i.e. subquotients of the natural number object of Eff, see [11] —; and PER is the intersection of the last two, the full subcategory of $\mathcal{E} f f$ on the $\neg \neg$-separated subquotients of the natural number object of $\mathcal{E f f}$, also known as "partial equivalence relations on $\mathbb{N}$ ", see [14]. As is shown in [5], this last is not the regular completion of $\mathcal{P R} \equiv \mathcal{P} \mathcal{A} s m_{0}$. A similar remark applies to $\mathcal{E} q u$ and $\left(\mathcal{T}_{o p_{0}}\right)_{\text {reg }}$ which are not equivalent - this corrects a hastily mistaken, happily irrelevant statement in [3].

\section{Groupoids}

Consider a category $\mathcal{C}$ with pullbacks. A groupoid $\mathbb{G}$ in $C$ is a graph $G_{1} \underset{d_{2}}{\stackrel{d_{1}}{\longrightarrow}} G_{0}$ of objects and arrows in $C$ together with three more arrows

$$
i: G_{0} \longrightarrow G_{1} \quad c: G_{1} \times_{G_{0}} G_{1} \longrightarrow G_{1} \quad s: G_{1} \longrightarrow G_{1}
$$

where

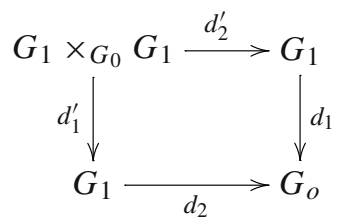

is a pullback in $\mathcal{C}$, such that

- the graph $G_{1} \underset{d_{2}}{\stackrel{d_{1}}{\longrightarrow}} \geq G_{0}$ with $i$ and $c$ is a category object in $\mathcal{C}$,

$-s$ is an involution which makes every arrow an isomorphism.

The notions of functor of groupoids in Cis obvious as well as that of natural transformation. It is straightforward to check that a functor between groupoids preserves the involution which makes every arrow an isomorphism.

We have already available a large number of examples as follows from the next property. 


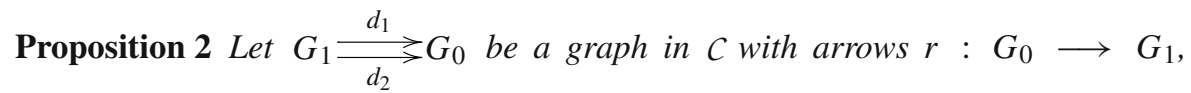
$t: G_{1} \times_{G_{0}} G_{1} \longrightarrow G_{1}$, and $s: G_{1} \longrightarrow G_{1}$ such that the diagrams

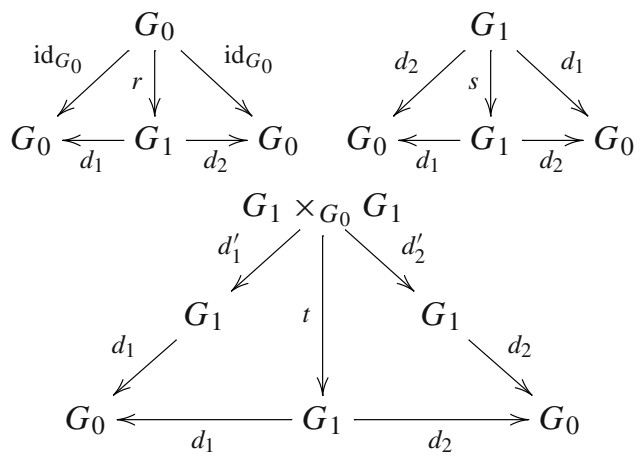

commute. If the pair $G_{1} \underset{d_{2}}{\stackrel{d_{1}}{\longrightarrow}} G_{0}$ is jointly monic, then

(i) the structure given by

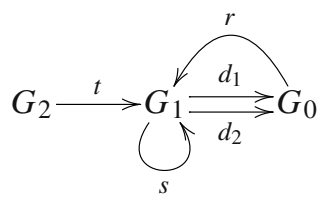

is a groupoid $\mathbb{G}$ in $\mathcal{C}$,

(ii) for any groupoid $\mathrm{H}$ in $\mathrm{C}$, a graph-homomorphism from the underlying graph $H_{1} \underset{e_{2}}{\stackrel{e_{1}}{\longrightarrow}} \geq H_{0}$ of $\mathbb{H}$ to $G_{1} \underset{d_{2}}{\stackrel{d_{1}}{\longrightarrow}} \geq G_{0}$ is also a functor from $\mathbb{H}$ to $\mathbb{G}$,

(iii) for any groupoid $\mathbb{H}$ in $\mathcal{C}$, let $\left(f_{1}, f_{0}\right)$ and $\left(g_{1}, g_{0}\right)$ be functors from the groupoid $\mathrm{H}$ to the groupoid $\mathbb{G}$. Then an arrow $a: H_{0} \longrightarrow G_{1}$ such that

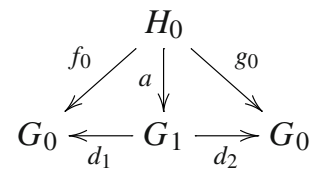

is a natural transformation from $\left(f_{1}, f_{0}\right)$ to $\left(g_{1}, g_{0}\right)$.

Proof Straightforward.

Corollary 1 Every subspatial equivalence span is a groupoid in Top p $_{0}$. Every representative of an arrow in $\mathcal{A}$ is a functor between the groupoids. 
Consider the interval-like groupoid

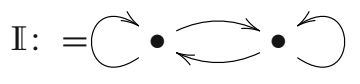

with the discrete topology. A natural transformation as in 2(iii) is the same as a functor $\mathbb{H} \times \mathbb{I} \longrightarrow \mathbb{G}$. Thanks to 1 , we may rephrase Corollary 1 as follows.

Theorem 1 The category Equ of equilogical spaces is the homotopical quotient of the category $\mathcal{A}$ of topological groupoids.

\section{2-Groupoids}

A similar case can be made for the effective topos. We prove in the following that it is the homotopical quotient of a category of higher groupoids in $\mathcal{P} \mathcal{A} s m$.

Consider a category $C$ with pullbacks. A 2-groupoid $\mathbb{G}$ in $C$ is a 2-graph

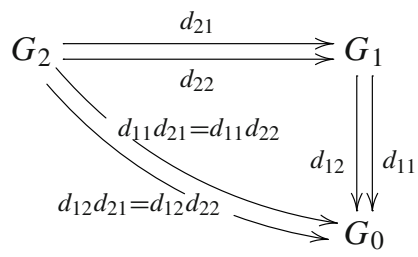

of objects and arrows in $C$ together with arrows

$$
\begin{aligned}
& i_{1}: G_{0} \longrightarrow G_{1} \quad c_{1}: G_{1} \times_{G_{0}} G_{1} \longrightarrow G_{1} \quad s_{1}: G_{1} \longrightarrow G_{1} \\
& i_{2}: G_{1} \longrightarrow G_{2} \quad c_{2}: G_{2} \times_{G_{1}} G_{2} \longrightarrow G_{2} \quad s_{2}: G_{2} \longrightarrow G_{2} \\
& c c_{2}^{\prime}: G_{2} \times_{G_{0}} G_{2} \longrightarrow G_{2} \quad q: G_{1} \longrightarrow G_{2}
\end{aligned}
$$

where
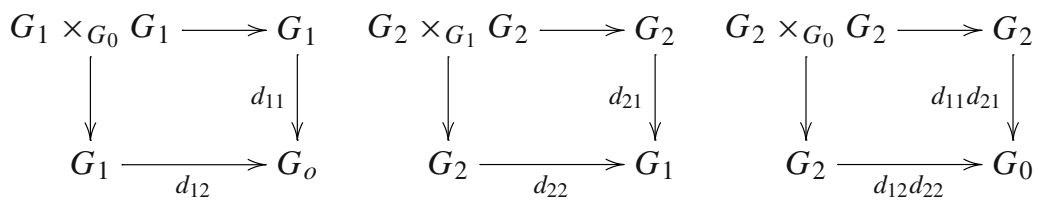

are pullbacks in $\mathcal{C}$, such that

- the 2-graph $G_{2} \underset{d_{22}}{\stackrel{d_{21}}{\longrightarrow}} G_{1} \underset{d_{12}}{\stackrel{d_{11}}{\longrightarrow}} G_{0}$ with $i_{1}, c_{1}, i_{2}, c_{2}, c_{2}^{\prime}$ is a 2-category object in $\mathcal{C}$

$-s_{1}$ is an involution which makes every 1 -arrow an equivalence via the pair of arrows given by $q$,

$-s_{2}$ is an involution which makes every 2-arrow an iso. 
The notions of 2-functor of 2-groupoids in $\mathcal{C}$ is obvious as well as that of 2transformation.

Consider the 2-category Grpd( $\mathcal{P} \mathscr{A} s m)$ of 2-groupoids in $\mathcal{P} \mathcal{A} s m$ with 2-functors and 2 -transformations. Clearly, the underlying graph of a 2-groupoid $\mathbb{G}$ of $\mathcal{P} \mathcal{A s m}$ is an equivalence span in $\mathcal{P} \mathcal{A} s m$, thus an object of $\mathcal{E} f f$. This extends directly to a functor $U: \operatorname{Grpd}(\mathscr{P} \mathcal{A} s m) \longrightarrow$ Eff.

Theorem 2 The functor $U: \operatorname{Grpd}(\mathcal{P A s m}) \longrightarrow$ Eff is essentially surjective.

Proof Consider an object in $\mathcal{E f f , ~ b y ~} 1$ we can assume without loss of generality that it

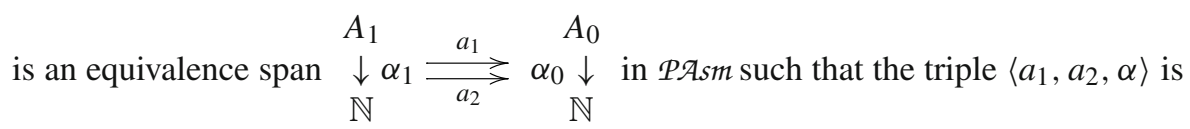
monic. Take the free dagger category on that graph in $\mathcal{P} A s m-$ by a dagger category we mean a category together with a involutive contravariant functor which is the identity $A_{0}$

$A^{\wedge}$ on objects. It consists of $\alpha_{0} \downarrow$ as objects of objects. The object of 1-arrows is $\alpha^{\wedge} \downarrow$ $\mathbb{N}$

where $A^{\wedge}$ consists of the zigzag paths in the graph $A_{1} \underset{a_{2}}{\stackrel{a_{1}}{\longrightarrow}} A_{0}$. By a zigzag path in the graph we mean a list which is either of the form $\langle x\rangle$ where $x \in A_{0}$ or

$$
\left\langle x_{0}, e_{1}, i_{1}, x_{1}, e_{2}, i_{2}, x_{2}, \ldots, x_{n}, e_{n+1}, i_{n+1}, x_{n+1}\right\rangle \text {, }
$$

where

$-x_{\ell} \in A_{0}$ for $0 \leq \ell \leq n+1$,

$-e_{\ell} \in A_{1}$ for $1 \leq \ell \leq n+1$,

$-i_{\ell} \in\{0,1\}$ for $1 \leq \ell \leq n+1$,

- for $0 \leq \ell \leq n$, if $i_{\ell}=0$, then $\left\langle x_{\ell}, x_{\ell+1}, e_{\ell+1}\right\rangle \in A_{1}$,

- for $0 \leq \ell \leq n$, if $i_{\ell}=1$, then $\left\langle x_{\ell+1}, x_{\ell}, e_{\ell+1}\right\rangle \in A_{1}$.

Intuitively, if one considers a triple $\left\langle x, x^{\prime}, e\right\rangle \in A_{1}$ as an edge $e$ from the source $x$ to the target $x^{\prime}$ in the graph $A_{1} \underset{a_{2}}{\stackrel{a_{1}}{\longrightarrow}} A_{0}$, then the zigzag

$$
\left\langle x_{0}, e_{1}, i_{1}, x_{1}, e_{2}, i_{2}, x_{2}, \ldots, x_{n}, e_{n+1}, i_{n+1}, x_{n+1}\right\rangle
$$

is a mixed-directional path of edges from the vertex $x_{0}$ to the vertex $x_{n+1}$ where each edge $e_{\ell}$ between $x_{\ell}$ and $x_{\ell+1}$ is marked with either 0 or 1: if the mark is $0, e_{\ell}$ goes from $x_{\ell}$ to $x_{\ell+1}$ in the original graph; if the mark is $1, e_{\ell}$ goes from $x_{\ell+1}$ to $x_{\ell}$. The function $\alpha^{\wedge}$ is defined by mapping a zigzag to the encoding of the list of its numerical components:

$$
\begin{aligned}
& \alpha^{\wedge}(\langle x\rangle):=\left\langle\left\langle 0, \alpha_{0}(x)\right\rangle\right\rangle \\
& \alpha^{\wedge}\left(\left\langle x_{0}, e_{1}, i_{1}, \ldots, x_{n}, e_{n+1}, i_{n+1}, x_{n+1}\right\rangle\right) \\
& \quad:=\left\langle\left\langle n+1,\left\langle\left\langle\alpha^{\wedge}\left(\left\langle x_{0}, e_{1}, i_{1}, \ldots, x_{n}\right),\left\langle\left\langle\left\langle e_{n+1}, i_{n+1}\right\rangle, a_{0}\left(x_{n+1}\right)\right\rangle\right\rangle\right\rangle\right\rangle\right\rangle .\right.\right.
\end{aligned}
$$


The structure of dagger category in $\mathcal{P A s m}$ is obvious, changing each $i_{\ell}$ with $\sigma\left(i_{\ell}\right)$ $A^{\text {凡 }}$

where $\sigma:\{0,1\} \longrightarrow\{0,1\}$ swaps 0 with 1 . The object of 2-arrows $\alpha^{-} \downarrow$ is formed $\mathbb{N}$

by taking the total relation on each 1-homset, where $A^{\wedge}:=A^{\wedge} \times{ }_{A_{0}} A^{\wedge}$. Explicitly, $A^{\Uparrow}$ consists of all pairs of zigzags

$$
\left\langle\left\langle x_{0}, e, \ldots, x_{n}\right\rangle,\left\langle x_{0}, e^{\prime}, \ldots, x_{n}\right\rangle\right\rangle
$$

between each two given vertices $x$ and $x^{\prime}$; clearly all 2-diagrams commute as there is at most one 2-arrow from an 1-arrow to another. In this way, the dagger functor becomes the involution which makes every 1 -arrow an equivalence. It is easy to see that that gives a 2-groupoid on the given span in $\mathcal{P} \mathcal{A} s m$ and that the functor $U$ takes it

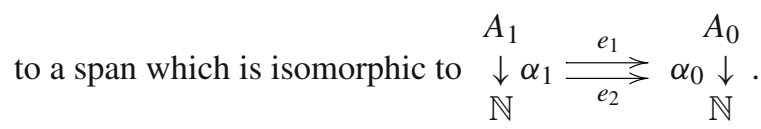

We shall refer to a 2-groupoid like that produced in the proof of 2 as a numeric 2 -groupoid as all edges are denoted by numbers. More precisely, it is a 2-groupoid $\mathbb{G}$ in $\mathcal{P} \mathcal{A} s m$ such that its underlying category in $\mathcal{P} \mathcal{A} s m$

$$
G_{1} \underset{d_{12}}{\stackrel{d_{11}}{\longrightarrow}} G_{0}
$$

is a free dagger category and $\mathbb{G}$ embeds, fully at level 2, into the 2-groupoid

$$
G_{0} \times G_{0} \times \mathbb{N} \times \mathbb{N} \frac{\pi_{123}}{\underset{\pi_{124}}{\longrightarrow}} G_{0} \times G_{0} \times \mathbb{N} \underset{\pi_{2}}{\stackrel{\pi_{1}}{\longrightarrow}} G_{0}
$$

where $\pi_{123}$ and $\pi_{124}$ are the projections deleting the fourth and third component, respectively.

Theorem 3 The functor $U: \operatorname{Grpd}(\mathcal{P A s m}) \longrightarrow$ Eff restricts to a homotopical quotient of the full subcategory $\mathcal{N}$ on the numeric 2-groupoids.

Proof Suppose that $\mathbb{G}$ and $\mathbb{H}$ are numeric groupoids. Since $\mathbb{G}$ is a free dagger category and all 2-diagrams commute in $\mathbb{H}$, it is easy to see that every arrow $[f]: U(\mathbb{G}) \longrightarrow$ $U(\mathbb{H})$ in $\mathcal{E} f f$ has a representative which is a 2 -functor $F: \mathbb{G} \longrightarrow \mathbb{H}$.

To see that the functor $U: \operatorname{Grpd}(\mathcal{P A} A s m) \longrightarrow$ Effrestricted to $\mathcal{N}$ is indeed a homotopical quotient, consider the interval-like groupoid I: it is the free dagger category on the graph in $\mathcal{P A s m}$ on $T+T$ with two (disjoint) nodes and a single edge $u$ connecting one with the other, with all possible 2-arrows. It is clearly a numeric 2-groupoid. Consider now two functors $F, F^{\prime}: \mathbb{G} \longrightarrow \mathbb{H}$ such that $U(F)=U\left(F^{\prime}\right)$; in other words, there is a map $k: G_{0} \longrightarrow H_{1}$ in $\mathcal{P} \mathcal{A} s m$ such that

$$
F_{0}=d_{11}^{\mathbb{H}} \circ k \quad \text { and } \quad F_{0}^{\prime}=d_{12}^{\mathbb{H}} \circ k .
$$


Note that the 1-category underlying the 2 -groupoid $\mathbb{G} \times \mathbb{I}$ is a retract of a free dagger category. Using $k$ to act on the generating arrow of II as follows

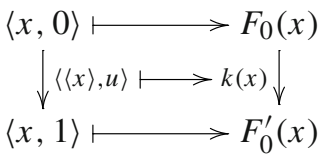

by freeness it is easy to obtain a functor $K: \mathbb{G} \times \mathbb{I} \longrightarrow \mathbb{H}$ which gives a homotopy from $F$ to $F^{\prime}$.

\section{References}

1. Bauer, A., Birkedal, L., Scott, D.S.: Equilogical spaces. Theor. Comput. Sci. 315(1), 35-59 (2004)

2. Birkedal, L., Carboni, A., Rosolini, G., Scott, D.S.: Type theory via exact categories. In: Pratt, V. (ed.) Proc. 13th Symposium in Logic in Computer Science, pp. 188-198. I.E.E.E. Computer Society, Indianapolis (1998)

3. Bucalo, A., Rosolini, G.: Sobriety for equilogical spaces. Theor. Comput. Sci. 546, 93-98 (2014)

4. Brown, R.: Elements of modern topology. McGraw Hill, USA (1968)

5. Carboni, A.: Some free constructions in realizability and proof theory. J. Pure Appl. Algebra 103, 117-148 (1995)

6. Carboni, A., Celia Magno, R.: The free exact category on a left exact one. J. Aust. Math. Soc. 33(A), 295-301 (1982)

7. Carboni, A., Freyd, P.J., Scedrov, A.: A categorical approach to realizability and polymorphic types. In: Main, M., Melton, A., Mislove, M., Schmidt, D. (eds.) Mathematical Foundations of Programming Language Semantics, vol 298 of Lectures Notes in Comput. Sci., pp. 23-42. Springer-Verlag, New Orleans (1988)

8. Carboni, A., Vitale, E.M.: Regular and exact completions. J. Pure Appl. Algebra 125, 79-117 (1998)

9. Freyd, P.J., Scedrov, A.: Categories, Allegories. North Holland Publishing Company, Amsterdam (1990)

10. Hyland, J.M.E., Johnstone, P.T., Pitts, A.M.: Tripos theory. Math. Proc. Camb. Philos. Soc. 88, 205-232 (1980)

11. Hyland, J.M.E., Robinson, E.P., Rosolini, G.: The discrete objects in the effective topos. Proc. Lond. Math. Soc. 60, 1-36 (1990)

12. Hofmann, M., Streicher, Th: The groupoid interpretation of type theory. Twenty-five years of constructive type theory (Venice. 1995), vol. 36 of Oxford Logic Guides, pp. 83-111. Oxford Univ. Press, New York (1998)

13. Hyland, J.M.E.: The effective topos. In: Troelstra, A.S., van Dalen, D. (eds.) The L.E.J. Brouwer Centenary Symposium, pp. 165-216. North Holland Publishing Company, Amsterdam (1982)

14. Hyland, J.M.E.: A small complete category. Ann. Pure Appl. Logic 40, 135-165 (1988)

15. Rosolini, G.: Equilogical spaces and filter spaces. Rend. Circ. Mat. Palermo 64(suppl.), 157-175 (2000)

16. Robinson, E.P., Rosolini, G.: Colimit completions and the effective topos. J. Symb. Logic 55, 678-699 (1990)

17. Scott, D.S.: Data types as lattices. SIAM J. Comput. 5(3), 522-587 (1976)

18. Scott, D.S.: A new category? Domains, spaces and equivalence relations. Manuscript (1996)

19. The Univalent Foundations Program: Homotopy Type Theory: Univalent Foundations of Mathematics. Institute for Advanced Study (2013) 